\title{
MINIMAL KINEMATIC BOUNDARY CONDITIONS FOR COMPUTATIONAL HOMOGENIZATION OF THE PERMEABILITY COEFFICIENT
}

\author{
Marek WOJCIECHOWSKI* \\ "Technical University of Lodz, Department of Civil Engineering, Architecture and Environmental Engineering, \\ Chair of Geotechnics and Engineering Structures, Al. Politechniki 6, 90-924 Łódź, Poland \\ mwojc@p.lodz.pl
}

received 16 March 2016, revised 15 September 2017, accepted 18 September 2017

\begin{abstract}
In the paper, computational homogenization approach is used for recognizing the macroscopic permeability from the microscopic representative volume element (RVE). Flow of water, at both macro and micro level, is assumed to be ruled by Darcy law. A special averaging constraint is used for numerical flow analysis in RVE, which allows to apply macroscopic pressure gradient without the necessity to use directly Dirichlet or Neumann boundary conditions. This approach allows arbitrarily shaped representative volumes and eliminates undesirable boundary effects. Generated effective permeability takes into account the structuring effects, what is an advantage over other homogenization methods, like self-consistent one.
\end{abstract}

Key words: Computational Homogenization, Permeability, RVE, Minimal Kinematic Boundary Conditions

\section{INTRODUCTION}

In broad class of disordered porous materials with clear matrix-inclusions internal structure, the flow of fluid can be described by Darcy equation at both, micro and macro level. This is for example the case of concrete and sand-clay soils. In order to determine the macroscopic response with accurate account for microstructural characteristics and evolution, computational homogenization strategy can be exploited (see Geers et al., 2001, Feyel, 2003; Kousnietsova et al., 2010). When using this micro macro strategy there is actually no necessity to define homogenized macroscopic constitutive equations. Instead, the constitutive behavior at macroscopic integration points is determined by averaging the response of the deforming microstructure. This enables a straightforward application of the method to geometrically and physically non-linear problems, making it a particularly valuable tool for the modeling of evolving non-linear heterogeneous microstructures under complex macroscopic loading paths.

Computational homogenization needs, at each macroscopic integration point, a microscopic representative volume to be defined and boundary value problem (BVP) to be solved. One of the concerns in homogenization theory is the kind of boundary conditions (BCs) which should be applied to this BVP. Recently, new concept in this area has been proposed, called minimal kinematic boundary conditions (see Mesarovic S.D. and Padbidri J., 2005; Inglis at al., 2008; Wojciechowski M. and Lefik M., 2016). This approach consists in applying special averaging constraint to the microscopic problem, instead of usual Dirichlet or Neumann boundary conditions. This allows for arbitrary shapes of RVE and eliminates undesirable boundary effects which may appear when e.g. periodic BCs are applied.

In the paper we use the computational approach for recognizing the macroscopic permeability from the microscopic representative volume element (RVE), which takes into account propor- tions, arrangement and shape of the material constituents. In the following, the homogenization scheme is formulated, the solution by finite element method is presented and the illustrative example is shown. Paper ends with some discussion and conclusions.

\section{HOMOGENIZATION FRAMEWORK}

Let's consider microstructurally complex porous material for which a representative volume element (RVE) $\Omega$ can be defined. In case of laminar flow, local flux $u_{i}$ in the RVE is given by Darcy equation (skipping source terms):

$u_{i}=-\frac{k_{i j}}{\gamma} p_{, j}$

where $p_{, j}$ : is a pressure gradient, $k_{i j}$ is a permeability tensor depending on the position in RVE (in velocity units) and $\gamma$ is a specific weight of the fluid. Averages of the microscopic fluxes and pressure gradients over domain $\Omega$ are given by:

$P_{, j}=\frac{1}{\Omega} \int_{\Omega} p_{, j} d \Omega$,

$U_{i}=\frac{1}{\Omega} \int_{\Omega} u_{i} d \Omega$

These values are assumed to be related via the effective permeability tensor $K_{i j}$, such that:

$U_{i}=-\frac{K_{i j}}{\gamma} P_{, j}$

The above equation describes macroscopic behavior of the composite. The fundamental concept from which the existence and consistency of the above averages can be derived is the Hill 
macrohomogeneity condition (Hill, 1965), which in case of Darcy flow is written as (Du X. and Ostoja-Starzewski M., 2006):

$$
\int_{\Omega} u_{i} p_{, i} d \Omega=\frac{1}{\Omega} \int_{\Omega} u_{i} d \Omega \int_{\Omega} p_{i} d \Omega
$$

\section{MACRO}

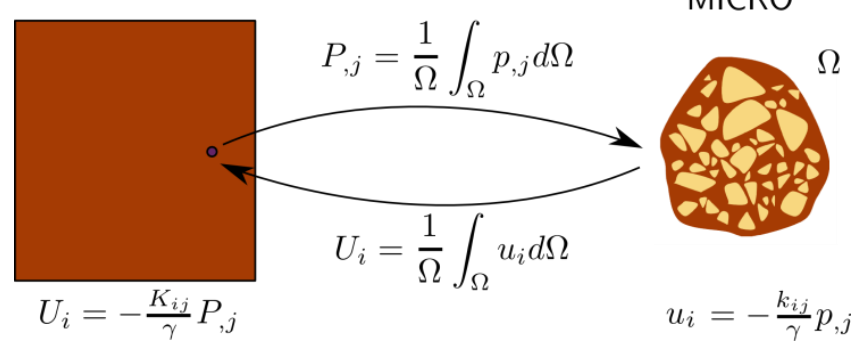

Fig. 1. Scheme of the computational homogenization for Dacy flow

Homogenization problem considered here is formulated as follows: find solution $p$ of the compatibility equation $u_{i, i}=0$ defined on $\Omega$, subject to some macroscopic pressure gradient $P_{j}$ in such a way that equation (2) is fulfilled. From this solution microscopic $u_{i}$ and macroscopic $U_{i}$ and $K_{i j}$ are then derived (see Figure 1 for homogenization scheme). The above can be viewed as a problem of minimization of total potential energy (see again Du X. and Ostoja-Starzewski M., 2006) with additional averaging constraint:

$\min _{p}\left[\int_{\Omega}-p_{, i} \frac{k_{i j}}{\gamma} p_{, j} d \Omega\right] \quad$ subject to

$-\Omega P_{, k}+\int_{\Omega} p_{, k} d \Omega=0$

which can be converted to unconstrained minimization by means of Lagrange multipliers $\lambda_{k}$ (Bertsekas, 1982):

$$
\min _{p}\left[\int_{\Omega}-p_{, i} \frac{k_{i j}}{\gamma} p_{, j} d \Omega+\lambda_{k}\left(-\Omega P_{, k}+\int_{\Omega} p_{, k} d \Omega\right)\right] .
$$

\section{FINITE ELEMENT METHOD FORMULATION}

Solutions $p$ of the above homogenization problem have to belong to the Sobolev functional space $H^{1}(\Omega)$ (at least), and this is assured by piecewise polynomial base functions used in FEM discretizations (see e.g. Babuška I. and Strouboulis T., 2001; Brenner S.C. and Scott R., 2007). Let's assume the domain $\Omega$ is discretized into $e=1, \ldots, E$ elements such that $\Omega=\Omega_{1} \cup \ldots \cup$ $\Omega_{E}=\sum_{e} \Omega_{e}$. Let's assume also, that each element has $n=$ $1, \ldots, N$ nodes in which the solution will be known. Pressures and its spatial derivatives inside elements are then given by linear combinations:

$p_{e}(\mathbf{x})=\phi_{e n}(\mathbf{x}) p_{e n}$,

$p_{e, i}(\mathbf{x})=\phi_{e n, i}(\mathbf{x}) p_{e n}$

where: $p_{e n}$ are the nodal pressures and $\phi_{e n}(\mathbf{x})$ are element interpolation functions defined on global Cartesian coordinates. For numerical integration pressure values at Gauss points inside elements need to be known. If number of these points per element is marked as $g=1, \ldots, G$, then arrays of pressures and their derivatives for the discretized domain are written as:

$p_{e g}=\phi_{\text {egn }} p_{e n}$,
$p_{\text {egi }}=\phi_{\text {egni }} p_{\text {en }}$

where: $\phi_{e g n}, \phi_{e g n i}$ are appropriate arrays of interpolation functions and their derivatives. Minimization problem (7) can be now rewritten with discretized pressure field, replacing integrals with sums over elements and integration points:

$\min _{p}\left[\sum_{e} p_{e m}\left(-\sum_{g} w_{e g} \phi_{e g m i} \frac{k_{e g i j}}{\gamma} \phi_{e g n j}\right) p_{e n}\right.$
$\left.+\lambda_{k}\left(-\Omega P_{, k}+\sum_{e} \sum_{g} \phi_{e g n k} p_{e n}\right)\right]=$

$\min _{p}\left[\sum_{e} p_{e m} A_{e m n} p_{e n}\right.$

$\left.+\lambda_{k}\left(-\Omega P_{, k}+\sum_{e} B_{e n k} p_{e n}\right)\right]$

where: $k_{e g i j}$ is an array of permeability tensors at Gauss points and $w_{e g}$ are weights of numerical integration. Moving from Einstein notation to the usual matrix representation of the finite element interpolations give us:

$\min _{\mathbf{p}}\left[\sum_{e} \mathbf{p}_{e}^{\mathrm{T}} \mathbf{A}_{e} \mathbf{p}_{e}+\lambda^{\mathrm{T}}\left(-\Omega \mathbf{P}+\sum_{e} \mathbf{B}_{e}^{\mathrm{T}} \mathbf{p}_{e}\right)\right]$.

Finally, dropping the sum over elements (i.e. performing global assembling) we get:

$\min _{\mathbf{p}}\left[\mathbf{p}^{\mathrm{T}} \mathbf{A} \mathbf{p}+\lambda^{\mathrm{T}}\left(-\Omega \mathbf{P}+\mathbf{B}^{\mathrm{T}} \mathbf{p}\right)\right]=\min _{\mathbf{p}}[\Pi(\mathbf{p}, \boldsymbol{\lambda})]$

where: $\mathbf{p}$ is global vector of unknown pressures of the length $M$ ( $M$ - total number of nodes in discretization), $\mathbf{A}$ is a global linear operator of the size $M \times M, \mathbf{B}$ is a problem specific matrix of the size $M \times D(D$ - space dimension: 2 or 3$), \boldsymbol{\lambda}$ is a vector of unknown Lagrange multipliers of the length $D$, and $\mathbf{P}$ is a vector of known, macroscopic pressure gradient to be applied, also of length $D$. Solution of the problem is found by differentiation of the resulting potential $\Pi$ with respect to unknown $\mathbf{p}$ and $\boldsymbol{\lambda}$ and equating derivatives to 0 :

$\frac{\partial \Pi(\mathbf{p}, \boldsymbol{\lambda})}{\partial \mathbf{p}}=\mathbf{A} \mathbf{p}+\mathbf{B} \boldsymbol{\lambda}=\mathbf{0}$,

$\frac{\partial \Pi(\mathbf{p}, \boldsymbol{\lambda})}{\partial \boldsymbol{\lambda}}=\mathbf{B}^{\mathrm{T}} \mathbf{p}-\Omega \mathbf{P}=\mathbf{0}$.

The above is a system of linear equations:

$\left[\begin{array}{cc}\mathbf{A} & \mathbf{B} \\ \mathbf{B}^{\mathrm{T}} & \mathbf{0}\end{array}\right]\left[\begin{array}{l}\mathbf{p} \\ \boldsymbol{\lambda}\end{array}\right]=\left[\begin{array}{c}\mathbf{0} \\ \Omega \mathbf{P}\end{array}\right]$.

For this problem only minimal set of Dirichlet boundary conditions should be applied, without introducing any additional pressure gradient and flux. This is achieved simply by fixing pressure at certain level: $p=p_{0}$ at single, arbitrary boundary point of the domain (or at arbitrary boundary node of its discretization).

Macroscopic, effective permeability can be now calculated from the observation, that Lagrange multipliers $\lambda$ have interpreta- 
tion of macroscopic average flux, with minus sign, i.e.: $\boldsymbol{\lambda}=-\mathbf{U}$ (see Wojciechowski M. and Lefik M., 2016). Combining equations (13) and (14) give us then:

$$
\begin{aligned}
& \Omega \mathbf{P}=\left(\mathbf{B}^{\mathrm{T}} \mathbf{A}^{-1} \mathbf{B}\right) \mathbf{U}, \\
& \mathbf{U}=\Omega\left(\mathbf{B}^{\mathrm{T}} \mathbf{A}^{-1} \mathbf{B}\right)^{-1} \mathbf{P}
\end{aligned}
$$

and from equation (4) we get finally the effective permeability:

$K_{i j}=\mathbf{K}=-\gamma \Omega\left(\mathbf{B}^{\mathrm{T}} \mathbf{A}^{-1} \mathbf{B}\right)^{-1}$.

One should note that there is no direct dependence between effective permeability and macroscopic pressure gradient, thus the same result will be obtained for any $P_{, j}$. This means that explicit solution of the system of linear equations (15) is actually not needed. This will be true unless microscopic constitutive equation is nonlinear, i.e. unless operator $\mathbf{A}$ is pressure dependent. In addition one can observe, that equation (17) needs a kind of inversion of the operator $\mathbf{A}$ which might be costly for large number of the RVE discretization nodes (or even impossible). However, in these cases numerical differentiation can be used.

\section{NUMERICAL EXAMPLE}

Presented homogenization method for Darcy flow has been implemented in the frame of finite element package fempy (Wojciechowski, 2014). We performed calculations for exemplary two-dimensional representative volume of the sand-clay mixture (for permeability properties of such materials see e.g. Juang and Holtz, 1986; Chapuis, 1990; Revil and Cathles, 1999; Kacprzak, 2006; Boutin et al., 2011). RVE of the reference size about $5 \mathrm{~mm}$ is considered, with irregular boundary adjusted to the randomly generated grain distribution (see figure 2). Average particle size diameter has been chosen uniformly, in a random way, from the range $0.1-1 \mathrm{~mm}$. The shape of sand grains is also irregular. Volume ratio of grains is assumed to be equal to $G=0.4$. Sand grains are assumed to be impermeable. Matrix consists in saturated kaolin clay with the void ratio $e_{c}=1$. Following the results reported by Kacprzak (2006) and Boutin et al. (2011) (see e.g. paragraph 4.4 in this second reference) the permeability tensor for such clay paste is taken as $k_{c}=\left[\left[10^{-9}, 0\right],\left[0,10^{-9}\right]\right] \mathrm{m} / \mathrm{s}$. Also, specific weight for water $\gamma=9.807 \mathrm{kN} / \mathrm{m}^{3}$ is taken for calculations.
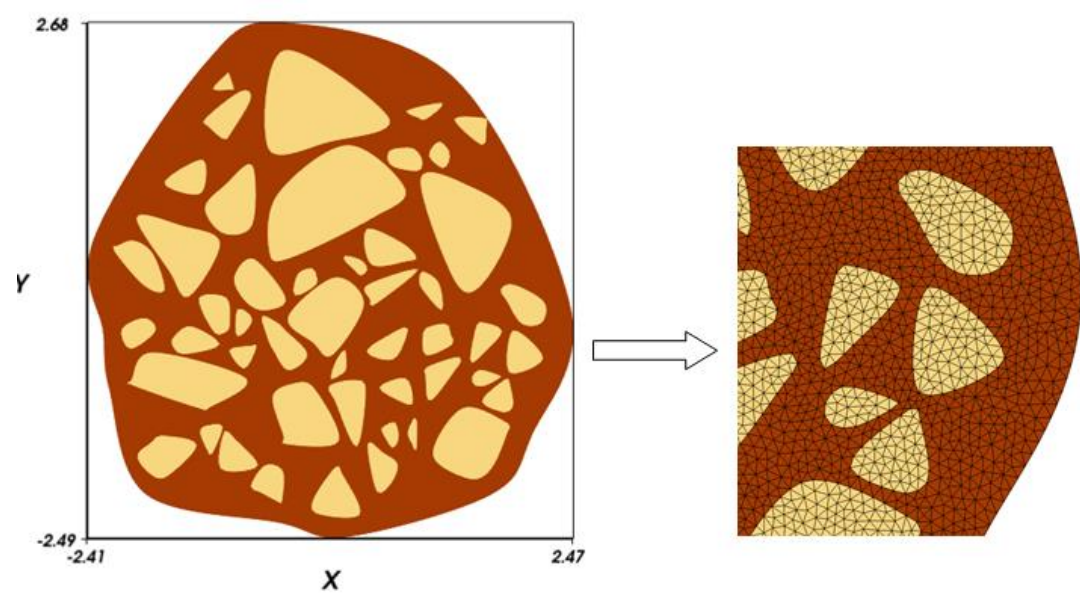

Fig. 2. Geometry of the representative volume element (dimensions in millimeters) and part of finite element discretization
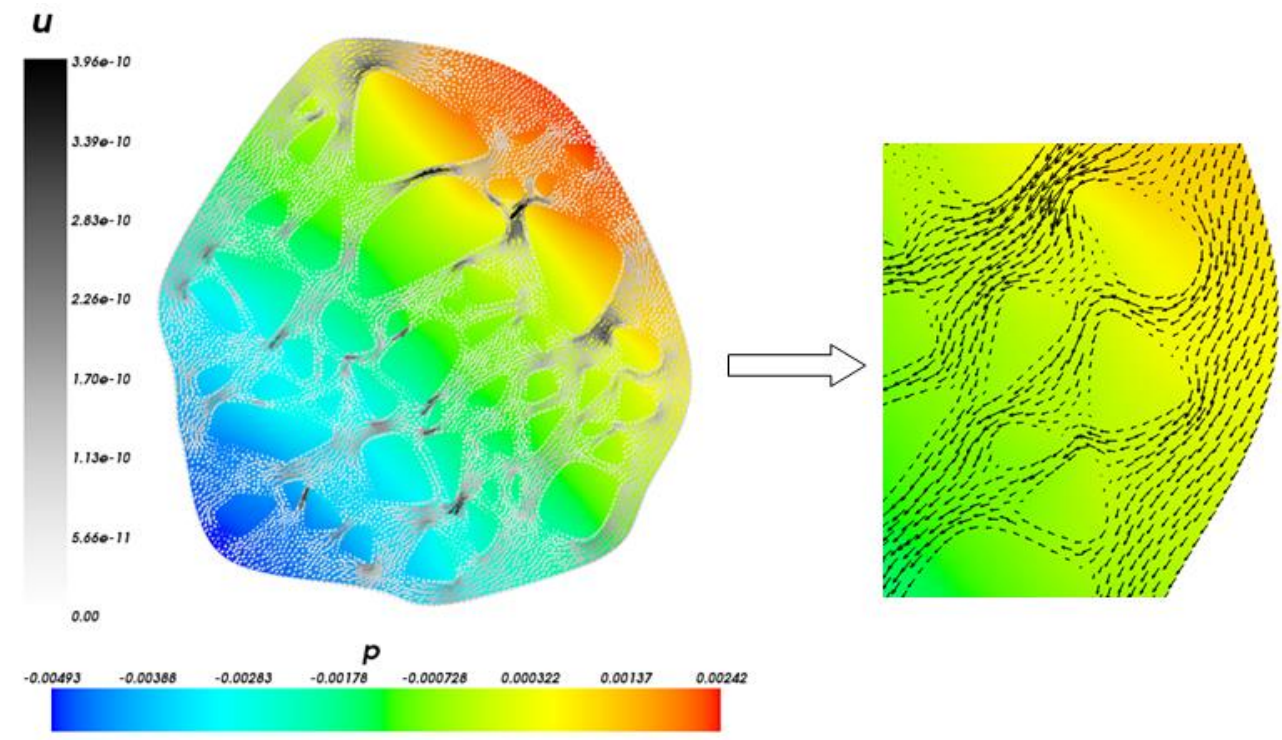

Fig. 3. Pressures $p[\mathrm{kPa}]$ and Darcy flux $u[\mathrm{~m} / \mathrm{s}]$ distribution inside the RVE after application of macroscopic pressure gradient. On the right hand side: zoom on the flux vectors. 
Geometry of the RVE has been discretized into 3-noded, triangular, linear finite elements. Very fine discretization (10647 nodes, 21003 elements) is used for better handling grain shapes, but also coarser mesh should provide acceptable results (it was not tested though). In this example the reference pressure has been taken as $p_{0}=0 \mathrm{kPa}$ at single boundary point of the discretization, and the macroscopic pressure gradient of the value $P_{, j}=[1,1] \mathrm{kPa} / \mathrm{m}$ is applied.

Results of the calculations are showed in Fig. 3. The flow occurs from top-right to bottom-left of the RVE, reflecting the applied macroscopic pressure gradient. The flux is zero inside the sand grains and varies in clay matrix taking the largest values in "bottlenecks" between grains. From this solution the macroscopic average flux is established from (3) as: $U_{i}=[-3.74$. $\left.10^{-11},-3.72 \cdot 10^{-11}\right] \mathrm{m} / \mathrm{s}$. Also the homogenized, macroscopic permeability tensor calculated from equation (17) is equal to:

$\mathrm{K}_{\mathrm{ij}}=\left[\begin{array}{cc}3.69 \cdot 10^{-10} & -3.22 \cdot 10^{-12} \\ -3.22 \cdot 10^{-12} & 3.68 \cdot 10^{-10}\end{array}\right] \mathrm{m} / \mathrm{s}$.

It's straightforward to verify, that equation (4) holds for these results.

\section{DISCUSSION OF RESULTS}

Macroscopic permeability obtained in numerical example is generally anisotropic, but it is almost equal in both Cartesian directions, i.e. $K=K_{11} \cong K_{22} \cong 3.7 \cdot 10^{-10} \mathrm{~m} / \mathrm{s}$. This indicates that the randomly generated RVE is appropriate for the considered problem - statistically isotropic at macroscale. The difference between $K_{11}$ and $K_{22}$, and also the small skew component $K_{12}$ should then tend to zero with increasing size of RVE. Comparisons to the oedometric laboratory tests reported by Kacprzak (2006) and Boutin et al. (2011) show, that computed permeability $K$ falls, as expected, into the range of variation of experimental results performed for similar volume ratio of sand grains and void ratio of clay paste.

Computational approach provides generally better approximation of the mixture permeability than simplified analytical methods. Self-consistent homogenization scheme for 2D case gives effective filtration coefficient equal to:

$K_{S-C}=k_{c} \cdot \frac{1-G}{1+G}$.

Exactly the same result will arise also from Mori-Tanaka homogenization scheme. For the parameters used in numerical example the above equation gives coefficient $K_{S-C}=4.3 \cdot 10^{-10} \mathrm{~m} / \mathrm{s}$, which is larger than numerically obtained $K$. This could be explained by the fact, that the structuring effects, i.e. irregular, elongated paths of water flow through the RVE, visible in figure 3, have been automatically taken into account in computational results, which is not the case in analytical result. One should note that the proposed numerical approach will produce reliable effective parameters for any $G$ and $k_{c}$, also if these parameters vary both at microscopic and macroscopic level. Conversely, in case of the mentioned analytical self-consistent method special extensions with additional grain distribution and concentration parameters have to be considered, in order to catch microscopic structuring effects (see e.g. Boutin, 2011).

\section{CONCLUSIONS}

Computational homogenization approach is an attractive method for dealing with Darcy flow in heterogeneous porous media. This is because of the possibility of taking into account local proportions, arrangements, shapes and permeability parameters of composite constituents. At every integration point of macroscopic problem such locally defined representative volume elements can be loaded with local macroscopic pressure gradients, in an average sense, to get the macroscopic flux vectors. In general, effective macroscopic permeability is actually not necessary to be explicitly established in this approach. However, as we have showed, obtaining it is quite straightforward and reliable. In numerical example, the obtained effective permeability takes into account the structuring effects in the sand-clay mixture and this is an advantage in comparison to other homogenization methods.

In this paper only an illustrative example of single RVE analysis is presented, without actual macroscopic problem, as the main goal was presentation of the computational approach. Also, no special analysis is performed for establishing the adequate size and discretization of the RVE. Instead we relied on large representative volume with fine finite elements. It should be emphasized, that the shape of RVE used in this paper is rather unusual in numerical homogenization, where square or box shapes are commonly used, for easy application of linear or periodic kinematic BCs (pressure gradients in this case). Irregular shape of RVE is possible thanks to the novel method of application of macroscopic pressure gradient. The influence of RVE shape on homogenization results is however an open topic and should be addressed in future works.

\section{REFERENCES}

1. Babuška I., Strouboulis T. (2001), The finite element method and its reliability, Clarendon Press, Oxford.

2. Bertsekas D.P. (1982), Constrained optimization and Lagrange multiplier methods, Athena Scientific, Belmont, Massachusetts.

3. Boutin C., Kacprzak G., Thiep D. (2011), Compressibility and permeability of sand-kaolin mixtures. Experiments versus non-linear homogenization schemes, International Journal for Numerical and Analytical Methods in Geomechanics, 35(1), 21-52.

4. Brenner S.C., Scott R. (2007), The mathematical theory of finite element methods (Vol. 15), Springer Science \& Business Media.

5. Chapuis R.P. (1990), Sand-bentonite liners: predicting permeability from laboratory tests, Canadian Geotechnical Journal, 27(1), 47-57.

6. Du X., Ostoja-Starzewski M. (2006), On the size of representative volume element for Darcy law in random media, in Proceedings of the Royal Society A: Mathematical, Physical and Engineering Science, 462(2074), 2949-2963, The Royal Society.

7. Feyel F. (2003), A multilevel finite element method (FE 2) to describe the response of highly non-linear structures using generalized continua, Computer Methods in Applied Mechanics and Engineering, 192(28), 3233-3244.

8. Geers M.G.D., Kouznetsova V.G., Brekelmans W.A.M. (2001), Gradient-enhanced computational homogenization for the micromacro scale transition, Le Journal de Physique IV, 11(PR5), Pr5-145.

9. Hill R. (1965), Continuum micro-mechanics of elastoplastic polycrystals, Journal of the Mechanics and Physics of Solids, 13(2), 89-101.

10. Inglis H.M., Geubelle P.H., Matouš K. (2008), Boundary condition effects on multiscale analysis of damage localization, Philosophical Magazine, 88(16), 2373-2397. 
11. Juang, C.H., Holtz R.D. (1986), Fabric, pore size distribution, and permeability of sandy soils, Journal of Geotechnical Engineering, 112(9), 855-868.

12. Kacprzak G. (2006), Etude du comportement mécanique des mélanges sable/argile, PhD ENTPE/INSA, Lyon.

13. Kouznetsova V.G., Geers M.G.D., Brekelmans W.A.M. (2010), Computational homogenisation for non-linear heterogeneous solids, Multiscale Modeling in Solid Mechanics: Computational Approaches, 3, 1-42.
14. Mesarovic S.D., Padbidri J. (2005), Minimal kinematic boundary conditions for simulations of disordered microstructures, Philosophical Magazine, 85(1), 65-78.

15. Revil A., Cathles L.M. (1999), Permeability of shaly sands, Water Resources Research, 35(3), 651-662.

16. Wojciechowski M. (2014) Fempy - finite element method in python, http://fempy.org, http://geotech.p.lodz.pl:5080/fempy, last access: August 2017.

17. Wojciechowski M., Lefik M. (2016), On the static nature of minimal kinematic boundary conditions, Engineering Transactions, 64(4), $581-587$ 